\title{
Characteristic Frequencies of the Human Blood Distribution System
}

\author{
Maja Bračič ${ }^{1}$, Peter V.E. McClintock ${ }^{2}$ and Aneta Stefanovska ${ }^{1}$ \\ ${ }^{1}$ Group of Nonlinear Dynamics and Synergetics, Faculty of Electrical Engineering, \\ University of Ljubljana, Tržaška 25, 1000 Ljubljana, Slovenia \\ ${ }^{2}$ Department of Physics, Lancaster University, Lancaster, LA1 \&YB, UK
}

\begin{abstract}
The same periodic components are demonstrated for different functions, and at different sites, of the human cardiovascular system. The wavelet transform is used to show that their frequencies and amplitudes are modulated, providing experimental evidence that the dynamics of blood distribution is governed by five almost periodic and mutually coupled processes.
\end{abstract}

\section{INTRODUCTION}

Living systems exhibit many phenomena that can be accounted for in terms of physics. It appears, for example, that Brownian ratchets may be responsible for macromolecular transport within cells [1,2], that stochastic resonance [3] facilitates information flow in neurons and mechanoreceptors [4], that spatiotemporal brain dynamics results from oscillatory behaviour of neural populations [5], and that peristalsis in vascular and intestinal smooth muscle can be well characterised in terms of coupled nonlinear oscillators [6]. Signals derived from the human cardiovascular system have been found to contain several periodic components [7-9], thereby raising the question of whether the blood distribution system and its regulatory mechanisms can also be described as a set of coupled oscillators [10]. We address this problem experimentally by extracting the characteristics of its dynamics from measured signals.

Following the pioneering work of Sayers [7] and Akselrod et al. [8], a range of spectral analysis techniques have been applied to blood pressure and heart rate variability (HRV) data, and rhythmic phenomena in these signals have been reported by several authors [9]. Periodic components have also been observed in peripheral blood flow signals measured by laser Doppler flowmetry [12-14]. Together with respiratory oscillations, spectral peaks around $0.1 \mathrm{~Hz}$ and below $0.05 \mathrm{~Hz}$ were revealed $[7,8,15]$ including a frequency component around $0.01 \mathrm{~Hz}[13,14]$. The question therefore arises as to whether these peaks are invariants of the blood distribution system as a whole?

CP502, Stochastic and Chaotic Dynamics in the Lakes: STOCHAOS,

edited by D. S. Broomhead, E. A. Luchinskaya, P.V. E. McClintock, and T. Mullin

(C) 2000 American Institute of Physics 1-56396-915-7/00/\$17.00 


\section{OBSERVATIONS}

To address this question, we have analysed several noninvasively measured signals of cardiovascular origin recorded simultaneously at different sites of the human body. Because our primary interest centres on the dynamics of blood distribution, the time-scale of observation was restricted to the average time ( $\sim 1$ minute) [11] of one cycle of blood through the system. The electrical activity of the heart (measured as an electrocardiogram, or ECG), blood pressure, respiration and peripheral blood flow were measured. The ECG was recorded by a standard instrument, with electrodes on both shoulders and one below the heart. Piezoeletric sensors were used to detect the blood pressure and respiratory movements of the thorax. The peripheral blood flow was measured by the laser-Doppler technique [14] on four different sites with similar density of vessels and network resistance.

Data were recorded for healthy young male subjects in repose. Each subject lay still on a bed, and was asked to relax: 15 minutes elapsed prior to the recording period of 20 minutes. The signals were digitized with 16 bit resolution: the ECG and blood pressure at a $400 \mathrm{~Hz}$ sampling rate and the other signals at $40 \mathrm{~Hz}$. The HRV signal, consisting of the times between consecutive heartbeats marked by the R-peaks, was derived from the ECG. The trend was removed from all signals. The

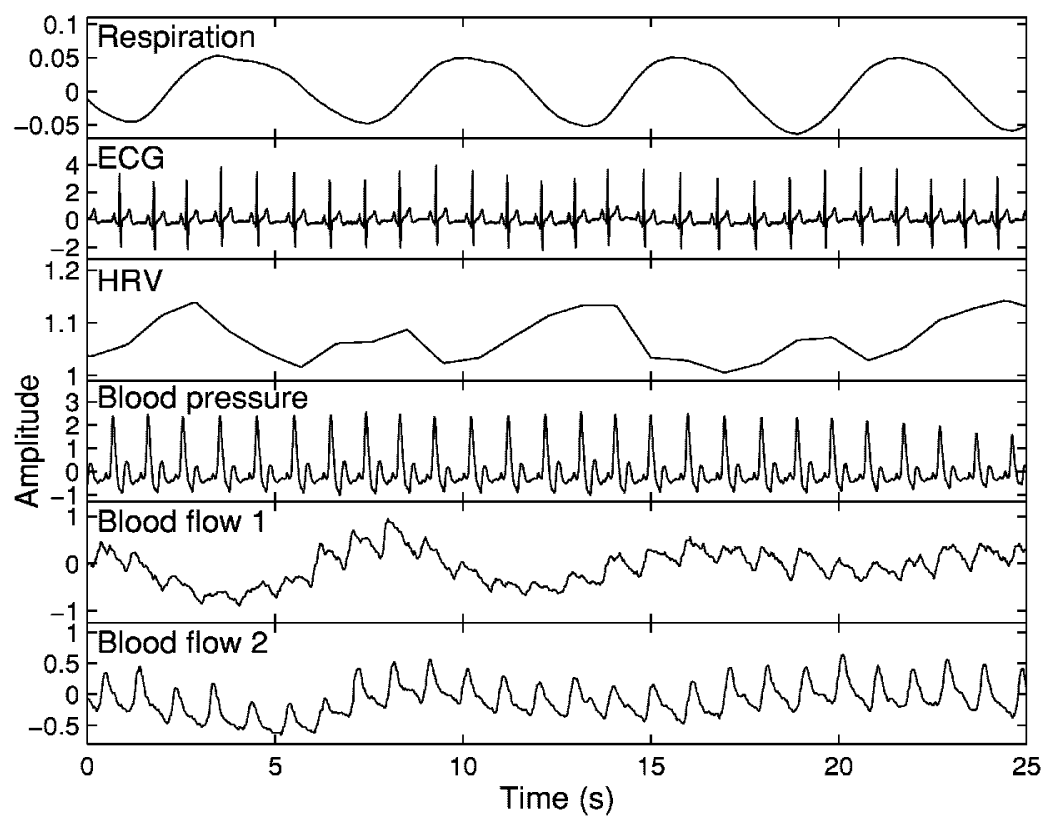

FIGURE 1. Samples of cardiovascular signals. 
moving average, calculated using a window of length $160 \mathrm{~s}$, shifted by $80 \mathrm{~s}$ along the signal, was interpolated and subtracted from the data. All signals except the ECG were resampled to $10 \mathrm{~Hz}$. Figure 1 presents the measured respiration, ECG, HRV, blood pressure, and peripheral blood flow on the right arm and right leg after preprocessing.

\section{TIME-FREQUENCY ANALYSIS}

Signals of cardiovascular origin, in particular the HRV signal, have been extensively analysed in the time and frequency domain, and in phase space. Transients, noise and nonstationarities, which are inherent to biological signals, restrict the application of phase space methods. Methods in the frequency domain, on the other hand, are less sensitive to noise and nonstationarities. However, there are two major difficulties related to the frequency analysis of such signals:

(i) time-variation of their characteristic frequencies, which demands an analysis in the time-frequency domain; and

(ii) the relatively broad frequency band within which characteristic peaks are expected, raising problem with respect to time and frequency resolution.

For these reasons the wavelet transform $[16,17]$, a time-frequency method providing for the necessary resolution at both high and low frequencies, was used. The wavelet transform is a scale-independent method. A chosen window function, the mother wavelet, is translated along the signal and also scaled - instead of a single window length, as in the conventional windowed Fourier method, all possible lengths can be used.

To detect the frequency content in a given time interval, Morlet [16] proposed a Gaussian window modulated by a sine wave. For cardiovascular signals we have chosen a sine wave with a basic frequency of $1 \mathrm{~Hz}$ [13]. In this case the wavelet transform estimates the contribution of the frequencies in a band around $f$ to the energy of a signal around time $t$. The bandwidth changes with scale, yielding a frequency resolution proportional to the observed frequency, $\Delta f=f / 2 \pi$. The time resolution is proportional to $1 / 2 f$. Thus it is possible to capture very different periodicities within a single signal. The time-frequency analysis is based on an approximation of the continuous wavelet transform [14]. This approach differs from multiresolution wavelet analysis, which has also been applied to cardiovascular signals [18] and uses a discrete transform with orthogonal basis functions.

The wavelet transform generates a 3-dimensional plot in the time-frequency plane. To simplify the comparison of measured signals in terms of their frequency content, however, we first consider the time-averaged wavelet transform. Application of this procedure to the data in Fig. 1 results in the spectra plotted in Fig. 2. It is immediately evident that there are several peaks in each spectrum and that they appear at similar, or in some cases even at exactly the same, frequencies. The widths of the peaks can largely be attributed to time variations of their characteristic frequencies, as we shall see. 


\section{CHARACTERISTIC FREQUENCIES}

The frequencies of the peaks in time-averaged wavelet transforms derived from 17 subjects are shown in Fig. 3. Between $0.0095 \mathrm{~Hz}$ and $1.6 \mathrm{~Hz}$ four peaks appear in the HRV and there are five in the blood flow signals for all subjects. It is evident that the peaks lie in distinct clusters: despite small differences between frequencies, each peak is located in the same range in all subjects. These five frequencies can be taken as characteristic of the blood distribution system. It is evident from Fig. 2 that the peak at $\sim 1.1 \mathrm{~Hz}$ corresponds to the heart rate, and that at $\sim 0.18 \mathrm{~Hz}$ to respiration. Lower frequency peaks are assumed to be of local origin, resulting from spatially distributed regulatory systems.

Oscillations of period $\sim 10 \mathrm{~s}$ in the blood pressure and HRV have been studied in connection with blood pressure regulatory mechanisms $[15,19]$. They are a manifestation of the myogenic activity of the smooth muscle cells of resistive vessels. These cells respond continuously to changes in intravascular pressure [20]. The

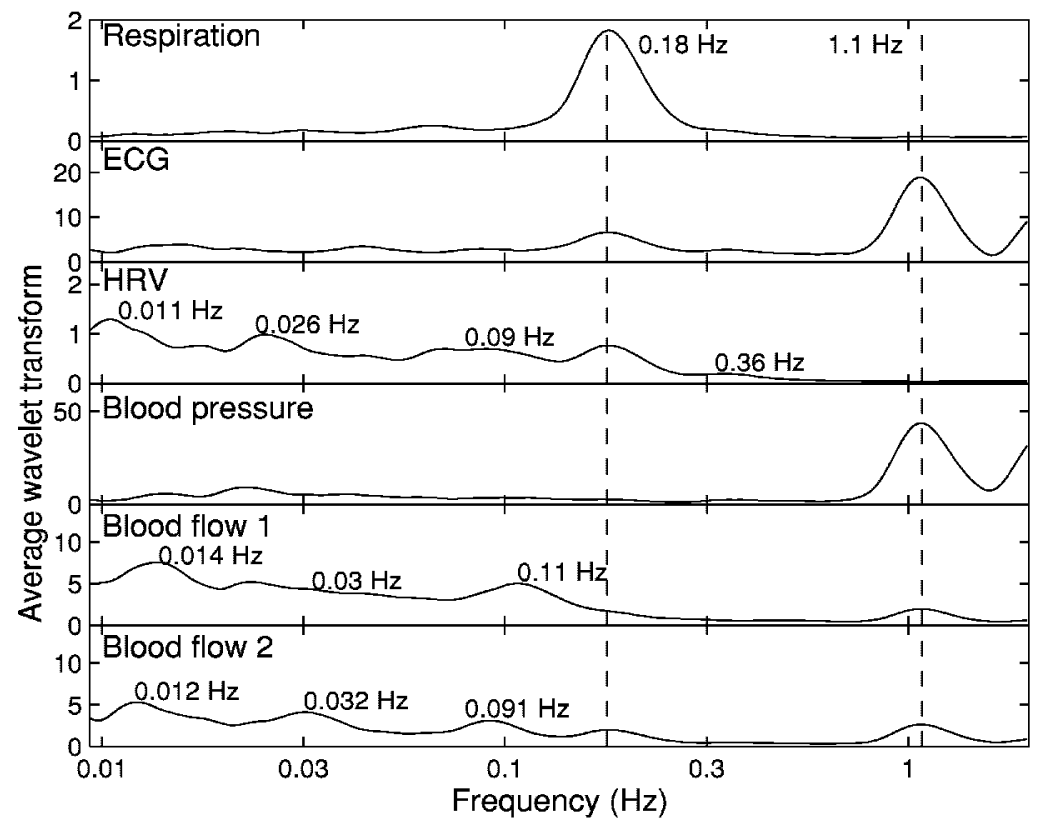

FIGURE 2. Time-averages of the wavelet transforms of signals presented in Figure 1. While the corresponding amplitudes may differ considerably, almost the same frequencies characterize all signals and can thus be taken as characteristic of the blood distribution system. The width of each peak is largely due to its time-variability, illustrated in detail in Figure 4 for one of the blood flow signals. 


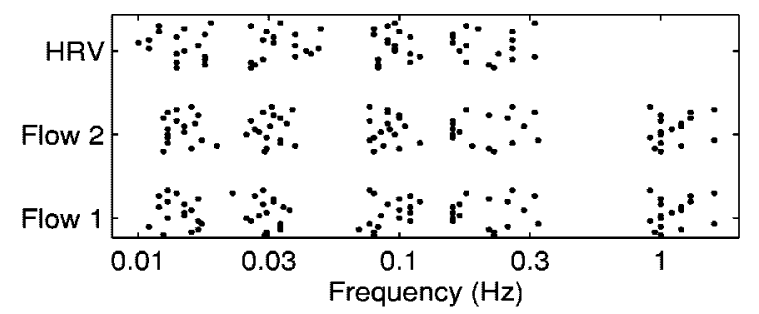

FIGURE 3. The positions of peaks in time-averaged wavelet transforms of the blood flow and HRV signals measured for 17 healthy subjects. The peak around $1 \mathrm{~Hz}$ is absent, by construction, from the HRV spectrum. The $y$-displacement of each point corresponds to a particular subject number, organised in the same order for all three signals.

corresponding peak at $\sim 0.1 \mathrm{~Hz}$ is clearly visible in both blood flow signals. The peak between $0.02 \mathrm{~Hz}$ and $0.04 \mathrm{~Hz}$ appears to be attributable to neurogenic regulation of the vessels' radii, since it has been found to disappear after denervation [21]. In addition, a peak near $0.01 \mathrm{~Hz}$ was found in all cardiovascular signals: it was recently shown $[22,23]$ to be related to endothelial activity, which controls the contraction and relaxation of smooth muscle cells.

\section{INTERACTIONS}

So far, by considering averaged spectra, we have ignored one dimension of the problem - the time. Segments of the full wavelet transform of blood flow in the time-frequency plane, plotted in Fig. 4 (left column), immediately reveal a new level of complexity and beauty in the system dynamics: the amplitudes and frequencies of the characteristic peaks all vary in time.

For the sake of clarity, the frequency axis is divided into five intervals, each containing one of the characteristic peaks. The time axes are adjusted to the variations of frequencies and amplitudes - for faster oscillations, a shorter time interval is used. Each peak is presented as a 3-dimensional plot (left), complemented by the time dependence of its frequency (middle) and amplitude (right).

The frequency variation of the heart-rate peak, at $\sim 1.1 \mathrm{~Hz}$ (Fig. 4a), although now derived from a peripherally measured signal, is highly correlated in time with the HRV signal (Fig. 1) and their frequency content is equal, nicely illustrating that all the cardiac periodicities are propagated as traveling waves. The frequencies and amplitudes of the other peaks, i.e., respiratory, myogenic, neurogenic and endothelial related (Fig. $4 \mathrm{~b}-\mathrm{e}$ ) are also modulated.

¿From Fig. 4 it is clear that much of the width of the peaks in the averaged spectra of Fig. 2 is attributable to the relatively slow variations in frequency. The fact that such signals are largely deterministic (as revealed e.g., by studies of their chaotic behaviour [26], analysis of surrogate data sets [27] and Lyapunov spectra [28]) 

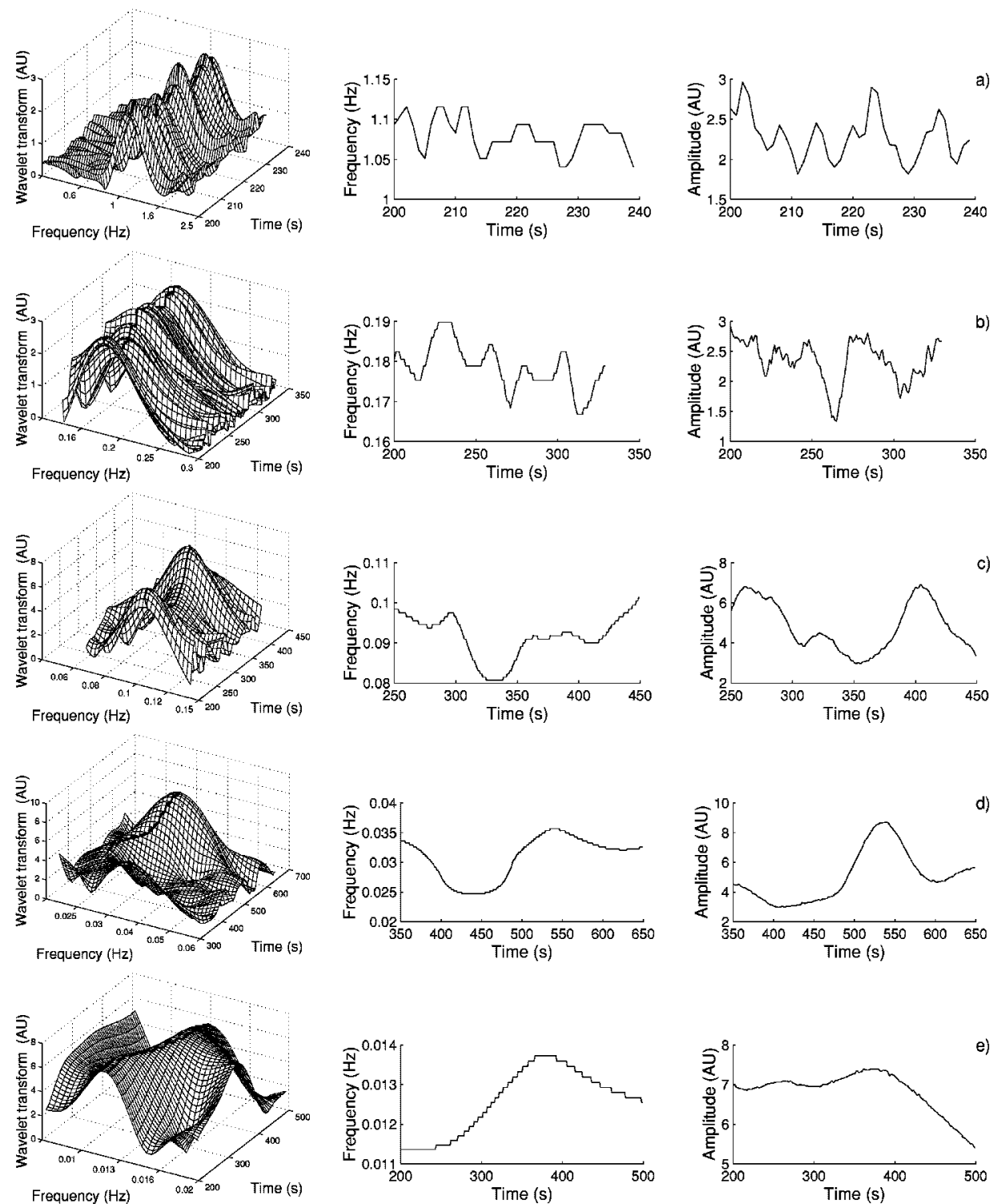

FIGURE 4. Segments of the wavelet transform of the blood flow signal in the time-frequency plane (left). On the right are plotted the amplitude and frequency variations of all five peaks. Each frequency and its amplitude are modulated by slower components. Discrete steps in the plots are due to finite frequency resolution. 
means that the amplitude/frequency wandering cannot in the main be attributed to external random fluctuations (noise), but must be inherent to the system itself.

These results suggest that the cardiovascular system as a whole, including the microcirculation, can usefully be treated as a single entity. The heart frequency and its variations are transmitted to the periphery by the blood motion, and are thus observable in peripheral blood flow or pressure. The lower-frequency (myogenic, neurogenic and endothelial-related) local regulatory mechanisms modulate the flow and in turn affect the variability of the heart rate. It is the continuous circulation of blood through the system of closed tubes that coordinates the local regulatory activity of each mechanism and evidently synchronizes it for much of the time (since, otherwise, its signature would not be resolved in the HRV signal). The existence of preferential phase and frequency-locking among spatially distributed components of a particular mechanism is manifested as the single almost periodic process that we observe at the macroscopic level. In terms of frequencies, it is irrelevant at which point we observe the system, or which function we choose to measure: each regulatory mechanism is reflected on every site, and can be detected in each cardiovascular function; its amplitude may, however, differ with respect to the function and the site of observation.

The results of Fig. 4 can be accounted for within the hypothesis that the blood distribution system is governed by a set of five coupled nonlinear oscillators [14]. The amplitude/frequency wandering then arises because of couplings between them, and it appears to reflect a complex combination of the possible interactions. It has long been acknowledged that coupling of the heart and respiration plays an essential role in the overall performance of the system [8]. Using bivariate cardirespiratory data it has recently been shown that episodes of synchronization can occur in a healthy system [29]. A similar analysis can be based on frequency traces of the kind discussed here. This would allow for a generalization of synchronisation methods: using the traces of lower peaks, their relationship to heart activity could

be analysed. Moreover, couplings between any of the systems could be treated in the same way using only a single measurement of the peripheral blood flow, or other cardiovascular signals. This is of particular importance for the lower frequency periodic components, which cannot be measured directly. In addition, the amplitude variations could also be taken into account.

The dynamics of the different physiological subsystems constituting the cardiovascular system are manifested on widely differing timescales. But in all cases not just the heart and respiration - they are characterised by slowly varying frequencies and amplitudes, processes that are evidently a characteristic feature of the dynamics of the blood distribution system as a whole.

\section{ACKNOWLEDGEMENTS}

This work was supported by the Slovenian Ministry of Science and Technology, INTAS, and the Engineering and Physical Sciences Research Council (UK). The 
authors would like to thank J. Petrovčič, R. Gush, M. Čenčur and D. Štajer and all the volunteers who participated in the measurements.

\section{REFERENCES}

1. Magnasco M.O., Phys. Rev. Lett. 71, 1477 (1993).

2. Bier M., Contemporary Phys. 38, 371 (1997).

3. Bulsara A., Chillemi S., Kiss L., McClintock P.V.E., Mannella R., Marchesoni F., Nicolis K. and Wiesenfeld K. Nuovo Cimento D 17, 653 (1995).

4. Wiesenfeld K., Pierson D., Pantazelou E., Dames C. and Moss F., Phys. Rev. Lett. 72, 2125 (1994).

5. Jirsa V.K. and Haken H., Phys. Rev. Lett. 77, 960 (1996).

6. Ermentrout G.B. and Kopell N., SIAM J. Math. Anal. 15, 215 (1984).

7. Sayers B. McA., Ergonomics 16, 16 (1973).

8. Akselrod S., Gordon D. Ubel F.A., Shannon D.C. Barger A.C. and Cohen R.J. Science 213, 220 (1981).

9. Di Renzo M., Parati G., Pedotti A., and Zanchetti A., Eds., Frontiers of Blood Pressure and Heart Rate Analysis, IOS Press, Amsterdam, 1997.

10. Stefanovska A., Strle S., Bračič M. and Haken H., Nonlinear Phenomena in Complex Systems, in print.

11. Schmidt R.F. and Thews G., Eds., Human Physiology, Springer-Verlag, 1989.

12. Hoffman U., Yanar A., Franzeck U.K., Edvards J.M., and Bollinger A., Microvasc. Res. 40, 293 (1990).

13. Bračič M. and Stefanovska A., Bull. Math. Biol. 60, 919 (1998).

14. Stefanovska A. and Bračič M., Contemporary Phys., 40, 31 (1999).

15. Hyndman B.W., Kitney R.I. and Sayers B.McA., Nature 233, 339 (1971).

16. Morlet J., In: Issues in Acoustic Signal/Image Processing and Recognition, edited by CH. Chen, NATO ASI Series, Vol.I, Springer-Verlag, Berlin, 1983.

17. Grossmann A. and Morlet J., SIAM J. Math. Anal. 15, 723 (1984).

18. Thurner S., Feurstein M.C. and Teich M.C., Phys. Rev. Lett. 80, 1544 (1998).

19. Kitney R.I., Fulton T., McDonald A.H. and Linkens D.A., J. Biomed. Eng. 7, 217 (1985).

20. Johnson P.C., NIPS 6, 41 (1991).

21. Kastrup J., Bühlow J. and Lassen N.A., Int. J. Microcirc.: Clin. Exp. 8, 205 (1989).

22. Kvernmo H.D., Stefanovska A., Kirkebøen K.A. and Kvernebo K., Microvasc. Res. $\mathbf{5 7}, 298$ (1999).

23. Stefanovska A., Bračič M. and Kvernmo H.D., IEEE Trans. Biomed. Eng., in print.

24. Task Force of the ECS and NASPE, Circulation 93, 1043 (1996).

25. Kaiser G., A Friendly Guide to Wavelets, Birkhäuser, Boston, 1994.

26. Poon C.S. and Merrill C.K. Nature 389, 492 (1997).

27. Stefanovska A. and Krošelj P., Open Sys. \& Information Dyn. 4, 457 (1997).

28. Bračič M. and Stefanovska A., Bull. Math. Biol. 60, 417 (1998).

29. Schäfer C., Rosenblum M.G., Abel H.H., Kurths J., Phys. Rev. E, 60, 857-870, (1999). 
Copyright $\odot 2003$ EBSCO Publishing 
Copyright of AIP Conference Proceedings is the property of American Institute of Physics and its content may not be copied or emailed to multiple sites or posted to a listserv without the copyright holder's express written permission. However, users may print, download, or email articles for individual use. 